\title{
RINGS WHICH ARE ALMOST POLYNOMIAL RINGS
}

\author{
BY
}

\section{PAUL EAKIN AND JAMES SILVER}

\begin{abstract}
If $A$ is a commutative ring with identity and $B$ is a unitary $A$ algebra, $B$ is locally polynomial over $A$ provided that for every prime $p$ of $A$, $B_{p}=B \otimes_{A} A_{p}$ is a polynomial ring over $A_{p}$. For example, the ring $Z\left[\left\{X / p_{i}\right\}_{i=1}^{\infty}\right]$, where $\left\{p_{i}\right\}_{i=1}^{\infty}$ is the set of all primes of $Z$, is locally polynomial over $Z$, but is not a polynomial ring over $Z$. If $B$ is locally polynomial over $A$, the following results are obtained. $B$ is faithfully flat over $A$. If $A$ is an integral domain, so is $B$. If $a$ is any ideal of $A$, then $B / a B$ is locally polynomial over $A / a$. If $p$ is any prime of $A$, then $p B$ is a prime of $B$. If $B$ is a Krull ring, so is $A$ and the class group of $B$ is is omorphic to the class group of $A$. If $A$ is a Krull ring and $B$ is contained in an affine doma in over $A$, then $B$ is a Krull ring. If $A$ is a noetherian normal domain and $B$ is contained in an affine ring over $A$, then $B$ is a normal affine ring over $A$. If $M$ is a module over a ring $A$, the content of an element $x$ of $M$ over $A$ is defined to be the smallest ideal $A_{x}$ of $A$ such that $x$ is in $A_{x} M$. A module is said to be a content module over $A$ if $A_{x}$ exists for every $x$ in $M . M$ is a content module over $A$ if and only if arbitrary intersections of ideals of $A$ extend to $M$. Projective modules are content modules. If $B$ is locally polynomial over a Dedekind domain $A$, then $B$ is a content module over $A$ if and only if $B$ is Krull.
\end{abstract}

Introduction. Let $Z$ denote the integers, $X$ an indeterminate over $Z$ and $\left\{p_{i}\right\}_{i=1}^{\infty}$ the set of prime numbers. Set $R=Z\left[\left\{X / p_{i}\right\}_{i=1}^{\infty}\right]$. Although the ring $R$ is neither noetherian nor Krull, as a $Z$-algebra it is locally indistinguishable from a polynomial ring in one variable over $Z$. That is, if $(p)$ denotes a prime ideal of $Z$ then $R_{p}=R \otimes_{z} Z_{(p)}=Z_{(p)}[X / p]$. In our terminology $R$ is locally a polynomial ring over $Z$.

Given a ring $A$ and an $A$-algebra $B$, we say that $B$ is locally a polynomial ring over $A$ if for every prime $p \subset A, B_{p}=B \otimes_{A} A_{p}$ is a polynomial ring over $A_{p}$. The above ring gives an example of a locally polynomial ring which is not a polynomial ring.

In this paper we examine the relationship between a ring $R$ and an algebra which is locally polynomial over $R$. We are particularly interested in deciding which properties of polynomial rings extend to this situation. This article is divided into three sections. The first section contains general results on locally polynomial rings. We prove that if $B$ is locally polynomial over $A$, then $B$ is faithfully

Presented to the Society, March 27, 1972; received by the editors December 13, 1971. AMS (MOS) subject classifications (1970). Primary 13A15, 13B20, 13B25, 13E05, 13F05, 13F 10, 13F 15, 13F20, 13G05, $13 \mathrm{H} 99$. 
flat over $A$. This yields the corollary that a locally polynomial ring over an integral domain is again a domain. We then observe that if $R$ is locally polynomial over a ring $A$ and $\mathscr{U}$ is an ideal of $A$, then $R / \mathfrak{U}$ is locally polynomial over $A / \mathscr{U}$. As an immediate corollary to these facts we show that primes of the ground ring extend to primes of the locally polynomial ring.

It is easily seen that if the ground ring is a domain then the number of indeterminates does not depend upon the prime at which we localize. This holds true for some more general classes of rings but not in general. In fact, for any chosen cardinal number $\alpha$, we give a method for constructing a ring $A$ and a locally polynomial ring $R$ over $A$ such that for every cardinal $\sigma$ between 0 and $\alpha$, there is a prime $p_{\sigma}$ of $A$ such that the generating set of $R_{p_{\sigma}}$ over $A_{p_{\sigma}}$ has cardinality $\sigma$.

$\S$ II is devoted to extending the concept of content from polynomial rings to locally polynomial rings. In fact, much of this can be done in the more general setting of modules over a ring. Where possible, we have maintained this setting. For a module $M$ over a ring $R$, the content of an element $x \in M$ is defined to be the smallest integral ideal $A_{x}$ of $R$ such that $x \in A_{x} M$. We show that a necessary and sufficient condition for every element of $M$ to have content is that arbitrary intersections of integral ideals of $R$ extend to $M$. We observe that every projective module is a content module and give examples to show that the converse is not true. If $M$ is a flat content module over $R$ and $S$ is a regular multiplicative system of $R$, then $M_{S}=M \otimes_{R} R_{S}$ is a content module over $R_{S}$. Furthermore, for every $x \in M$, the content of $x \otimes 1$ over $R_{S}$ is the extension to $R_{S}$ of the content of $x$ over $R$. This yields the corollary that, if $M$ is a flat content module over $R, x \in M$, and $s$ is a regular element of $R$, then $A_{s x}=s \Lambda_{x}$.

Restricting our considerations to locally polynomial rings, we are able to generalize some of the theorems for content in polynomial rings. For example, if $R$ is locally polynomial over a domain $D$ and has content over $D$, then, if for every pair $f, g$ in $\left.R_{D-\{0}\right\}_{f g}=A_{f} A_{g}, D$ is Prüfer. We prove that, if $R$ is locally polynomial over a Dedekind domain $A$, then $R$ is Krull if and only if $R$ has content over $A$. To conclude this section, we show that, if $R$ is locally polynomial over a Krull domain $A$ and has content over $A$, then $R$ is Krull.

To date it seems that the only reference to "content modules" is in an article by $\mathrm{Ohm}$ and Rush [OR]. There they propose definitions for content modules and algebras and refer to a forthcoming article [OR, cma] on the subject. Their definition of content module is not the same as ours although the basic ideas are certainly identical. Moreover, when considering their definition, one would be naturally led to the one we have taken. At this writing [OR, cma] is not available: it is to be expected that there will be some duplication of results.

The third section contains results which are obtained by imposing finiteness conditions on the ground ring. If $R$ is a Krull domain which is locally polynomial 
over a Krull domain $D$ then the divisor class group of $R$ is isomorphic to that of $D$. The example at the beginning of this introduction shows that, in general, it is not true that a locally polynomial ring over a Krull ring need be Krull. We show that if $R$ is almost a polynomial ring over a Krull ring $A$ and is contained in an affine domain over $A$, then $R$ is affine over $A$. We examine the noetherian case and give some necessary and sufficient conditions for a locally polynomial ring to be noetherian if the ground ring is noetherian. If $A$ is a noetherian normal ring and $R$ is locally polynomial over $A$ and contained in an affine ring over $A$, then $R$ is a normal affine ring over $A$. This statement also holds if "pseudogeometric" is substituted for "normal". We are grateful to Bill Heinzer for the benefit of several stimulating conversations on this subject.

(1.0) Throught this paper, we shall assume that all rings are commutative rings with identity. All modules and algebras will be assumed to be unitary.

(1.1) Definition. If a ring $B$ is an algebra over a ring $A$, we say that $B$ is locally polynomial over $A$ provided that, for every prime $p$ of $A, B_{p}=B \otimes_{A} A_{p}$ is a polynomial ring over $A_{p}$.

(1.2) If $B$ is locally polynomial over $A$, then $B$ is faithfully flat over $A$.

Proof. For every maximal ideal $m$ of $A, B_{m}$ is faithfully flat over $A_{m}$. Thus $B$ is faithfully flat over $A$ by [B, p. 116].

If $\sigma: A \rightarrow B$ makes $B$ an $A$-algebra which is locally polynomial over $A$, then for any element $a \in A, \sigma^{-1}(\sigma(a) B)=(a)$ by [B, p. 50]. Thus if $a \neq 0$, then $\sigma(a) \neq 0$ and $\sigma$ is an injection. By identifying $A$ with the subring $\sigma(A)$ of $B$, we can assume that $A$ is contained in $B$. For the remainder of this paper, we will make this assumption.

When $A$ is contained in $B$, given a prime $p$ of $A, B_{p}$ can also be defined to be $B_{(A-p)}$.

(1.3) Suppose that $R$ contains a ring $A$ and that $p$ is a prime ideal of $A$. Let $M=A-p$. Then $R_{M} \cong R \otimes_{A} A_{p}$.

Suppose that $B$ is a polynomial ring over $A$. Then $B$ is certainly locally polynomial over $A$. There are however rings $A$ and $B$ such that $B$ is locally polynomial over $A$ but not a polynomial ring over $A$.

(1.4) Example. Let $A$ be a ring and $\mathcal{U}$ a nonprincipal, invertible ideal of $A$. If $X$ is an indeterminate over $A$, let $B=A[\mathfrak{V} X]$. Then since $\mathscr{O}$ will be locally principal, $B$ will be locally polynomial over $A$.

The next result is a corollary to (1.2). The proof is a direct application of the corollary in [B, p. 50]. 
(1.5) Corollary. If $B$ is locally polynomial over $A$ and $B$ is also noetherian, then $A$ is noetherian.

The converse to this corollary is not necessarily true.

(1.6) Example. Let $Z$ denote the ring of integers and $\left\{p_{i}\right\}_{i=1}^{\infty}$ the set of primes of $Z$. Set $R=Z\left[\left\{X / p_{i}\right\}_{i=1}^{\infty}\right]$ where $X$ is indeterminate over $Z$. For any prime $p_{i}$ of $Z, R_{p_{i}}=Z\left[X / p_{i}\right]$. Thus $R$ is locally polynomial over $Z . R$ is not however noetherian since the ideal $\left(\left\{X / p_{i}\right\}_{i=1}^{\infty}\right) R$ is not finitely generated.

(1.7) If $R$ is locally polynomial over an integral domain $D$, then $R$ is a domain.

Proof. Since $R$ is flat over $D$, we see by $[N, 1 \mathbf{r},(18.1)]$, that every element of $D$ is regular in $R$. Let $p$ be any prime of $D$. Then the multiplicative system $M=D-p$ must be regular in $R$. Hence when we localize $R$ at $M, R$ is embedded in $R_{M} \cdot R_{M}$ is a polynomial ring over the domain $D_{p}$. Hence it is a domain and so is $R$.

(1.8) Suppose that $R$ is locally polynomial over a ring $A$ and that $\mathcal{O}$ is an ideal of $A$. Then $R / \mathscr{U} R$ is locally polynomial over $A / \mathfrak{X}$.

Proof. This is a straightforward application of the permutability of residue class ring and quotient ring formation.

(1.9) If $B$ is locally polynomial over $A$ and $p$ is a prime of $A$, then $p B$ is a prime of $B$.

Proof. By (1.8) $B / p B$ is locally polynomial over the domain $A / p$ and by (1.7) $B / p B$ is a domain. Therefore $p B$ is prime.

For a ring $A$, we let spec $A$ represent the set of all prime ideals of $A$.

(1.10) If $R$ is locally polynomial over a domain $D$ then $R=\bigcap_{p \in \operatorname{spec} D} R_{p}$.

Proof. Clearly $R \subseteq \bigcap_{p \in \operatorname{spec} D} R_{p}$. Let $q$ be any prime of $R$. Then $q \cap D=p$ is a prime of $D$. If $S^{\prime}=D-p$ and $S=R-q$, then $S^{\prime} \subseteq S$. Hence $R_{p}=R_{S^{\prime}} \subseteq R_{S}$ $=R_{q}$. Thus $\bigcap_{p \in \operatorname{spec} D} R_{p} \subseteq R_{q}$ and $\bigcap_{p \in \operatorname{spec} D} R_{p} \subseteq \bigcap_{q \epsilon \operatorname{spec} R} R_{q}=R$.

(1.11) If $R$ is locally polynomial over a domain $A$, then, for any beight one prime $p$ of $R$, either $p \cap A=G$ is a height one prime of $A$ or $p \cap A=(0)$ and $p$ $=P \cap R$ for some beight one prime $P$ of $R_{A-\{0\}}$.

Proof. If $G \neq(0)$, let $q$ be a prime such that $(0) \subseteq q \subset \mathscr{G}$. Since $R$ is faithfully flat over $A,(0) \subseteq q R \subset \mathfrak{G} R \subseteq p$. Thus $q R=(0), \mathfrak{G} R=p$, and $\mathrm{ht}(\mathfrak{G})=1$. If $\xi=(0)$, then $A-\{0\}$ is a multiplicative system of $A$ which misses $p$. Therefore, $p R_{A-\{0\}}=P$ is a height one prime of $R_{A-\{0\}}$ and $P \cap R=p$.

We have already observed that locally polynomial rings are stable under cer- 
tain homomorphisms. We shall now see that they are stable under certain types of integral extensions.

(1.12) Let $R$ be a domain which is locally polynomial over a domain D. Let $L$ be the quotient field of $D$ and $K$ the quotient field of $R$. If $L^{\prime}$ is an algebraic extension of $L$, then $R^{*}$, the integral closure of $R$ in $K\left[L^{\prime}\right]$, is locally polynomial over $D^{*}$, the integral closure of $D$ in $L^{\prime}$.

Proof. Let $p^{*}$ be a prime of $D^{*}$ and set $p^{*} \cap D=p$. Since $p$ is a prime of $D$, $R_{p}=D_{p}\left[X_{p}\right]$ for a set $X_{p}$ which is algebraically independent over $D$ and contained in $R$. Now $X_{p}$ is also algebraically independent over $D^{*}$ and $D_{p^{*}}^{*}\left[X_{p}\right] \subset R_{p^{*}}^{*}$. Since $R_{p}^{*}$ is integral over $R_{p}=D_{p}\left[X_{p}\right], R_{p^{*}}^{*}$ is integral over $D_{p^{*}}^{*}\left[X_{p}\right]$. Since $D_{p^{*}}^{*}$ is integrally closed we have $D_{p^{*}}^{*}\left[X_{p}\right]=R_{p^{*}}^{*}$.

We now consider the question of whether the number of indeterminates is constant or whether it depends upon the prime at which we localize. The answer is obvious in case the ground ring is an integral domain.

(1.13) If $R$ is locally polynomial over a domain $D$ then the number of indeterminates is constant and is equal to the transcendence degree of $R$ over $D$.

Example (1.18) will show that this result is not true in the general ring case. We can however give a partial extension by use of the following proposition.

(1.14) Suppose that $B$ is locally polynomial over a ring $A$ and let $p \subseteq q$ be two primes in $A$. Suppose $B_{p}=A_{p}\left[\left\{X_{a}\right\}_{a \in \mathbb{Q}}\right]$ and $B_{q}=A_{q}\left[\left\{X_{\beta}\right\}_{\beta \in \mathbb{B}}\right]$ then the cardinality of $\mathbb{Q}$ is identical to that of $\mathbb{B}$.

Proof. We first remark that if $A=R\left[\left\{X_{\gamma}\right\}_{\gamma \in \Gamma}\right]$ is a polynomial ring over $R$ and $p$ is a prime of $R$ then there is the natural homomorphism

$$
A=R\left[\left\{X_{\gamma}\right\}_{\gamma \in \Gamma}\right] \rightarrow R / P\left[\left\{X_{\gamma}\right\}_{\gamma \in \Gamma}\right]
$$

and the cardinality of $\Gamma$ is equal to the transcendence degree of $A$ over $R / p$. With this in mind, in view of (1.8), we can pass to the situation $B / p B$ as a locally polynomial ring over $A / p$. Then the assertion follows from (1.13).

(1.15) Suppose that $B$ is locally polynomial over a ring $A$ and suppose that $A$ bas the property that given any pair $m, m^{\prime}$ of maximal ideals in $A$ there is a collec. tion of maximal ideals $m_{0}=m, m_{1}, \ldots, m_{n-1}, m_{n}=m^{\prime}$ and a collection $p_{1}, \ldots$, $p_{n}$ of prime ideals of $A$ such that for every $i=1, \cdots, n, p_{i} \subseteq m_{i-1} \cap m_{i}$. Then the number of indeterminates is constant.

(1.16) Example. Suppose that $\Lambda=A_{1} \oplus A_{2}$ where $A_{1} \neq 0$ and $A_{2} \neq 0$. Let $R=A_{1}[X, Y] \oplus A_{2}[Z]$ where $X, Y$ and $Z$ are indeterminates over $A_{1}$ and $A_{2}$ respectively. The maximal ideals of $\Lambda$ are of the form $m_{1} \oplus A_{2}$ and $A_{1} \oplus m_{2}$ 
where $m_{1}$ and $m_{2}$ are maximal ideals of $A_{1}$ and $A_{2}$ respectively. Then $R_{\left(m_{1} \oplus A_{2}\right)}$ $\simeq A_{1 m_{1}}[X, Y] \simeq A_{\left(m_{1} \oplus A_{2}\right)}[X, Y]$ and $R_{\left(A_{1} \oplus m_{2}\right)} \simeq A_{2 m_{2}}[Z] \simeq A_{\left(A_{1} \oplus m_{2}\right)}[Z]$. Hence $R$ is locally polynomial over $A$ in a nonconstant number of indeterminates.

The obvious question is whether this is the only way the number of indeterminates can vary. To a limited extent this is true. The next result shows that a ring with only a finite number of height 0 primes must either satisfy (1.15) or be a nontrivial direct sum.

(1.17) Suppose that $A$ is a ring with only a finite number of beight 0 primes, $p_{1}, \cdots, p_{n}$ such that for some $k, 1<k<n, p_{i}+p_{j}=A$ for every pair $i, j, 1 \leq i$ $\leq k \leq j \leq n$. Then $A$ is a nontrivial direct sum.

This follows from a standard argument showing that one can lift idempotents modulo the nil radical.

The general situation is, however, more complicated. The example which follows gives a method for constructing a ring which is not a direct sum and over which we can construct a locally polynomial ring which has the property that by localizing at various primes of the ground ring every cardinality of indeterminates will appear up to any desired cardinality.

(1.18) Example. Let $\alpha$ be any cardinal number and let $W$ be the set of all ordinal numbers less than $\alpha$. Order $X^{\prime}=W \times[0,1)$ lexicographically and let $X=$ $X^{\prime} \cup\{a\}$. Order $X$ by setting $x<\alpha$ for every $x$ in $X^{\prime}$. Give $X$ the order topology. Let $A$ be the ring of continuous real valued functions on $X$. Since $X$ is connected, the ring $A$ is not a direct sum [GJ, 1B]. Furthermore, the fact that $X$ is compact implies that the maximal ideals of $A$ are precisely the fixed ideals $m_{x}=\{f: f(x)$ $=0\}$ where $x$ varies over all points in $X[\mathbf{G J},(4.8)]$. For each $\sigma \in W$, let $\left\{x_{n, \sigma}\right\}_{n=1}^{\infty}$ be a sequence of points in $\{x:(\sigma, 0)<x<(\sigma+1,0)\}$ such that for every open set $U$ containing $(\sigma+1,0)$ there is some $m$ such that $x_{n, \sigma} \in U$ for all $n \geq m$. Let $f_{n, \sigma}$ be a continuous function on $X$ which is never zero on $\left\{x: x<x_{n, \sigma}\right\}$ and identically zero on $\left\{x: x \geq x_{n, \sigma}\right\}$. Let $W^{\prime}=W \cup\{a\}$ and let $\left\{Z_{\sigma}: \sigma>0, \sigma \in W^{\prime}\right\}$ be an algebraically independent set over $A$. Take $Z_{0}=0$ and let $R^{\prime}=A\left[\left\{Z_{\sigma}: \sigma \in W^{\prime}\right\}\right]$. Let $\mathcal{U}$ be the ideal in $R^{\prime}$ generated by the set $\left\{f_{n, \sigma}\left(Z_{\sigma}-Z_{\sigma^{\prime}}\right): n=1,2, \ldots, \sigma^{\prime}>\sigma\right\}$ and $R=R^{\prime} / \mathcal{U}$. Since $\mathscr{U} \cap A=0, A$ can be considered a subring of $R, R$ is locally polynomial over $A$ and for every $\sigma \in W^{\prime}$, the cardinality of the set of indeterminates for $R_{m(\sigma, 0)}$ over $A_{m(\sigma, 0)}$ is $\sigma$.

(i) The space $X$ is compact and connected. (See [GJ, p. 262, Example 16-H].)

(ii) $R$ is locally polynomial over $A$. For any $x \in X, R_{m_{x}}=R_{m_{x}}^{\prime} / a R_{m_{x}}^{\prime}$ by the permutability of residue class and quotient ring formation. Suppose $X=(\sigma, r)$ for some $\sigma \in W$ and some $r \in[0,1]$. For all $\sigma^{\prime \prime}<\sigma, f_{n, \sigma}=0$ on some open interval containing $x$. Hence $f_{n, \sigma^{\prime \prime}}$ is in the kernel of the canonical map from $R^{\prime}$ 
to $R_{m_{x}}^{\prime}$ for all $\sigma^{\prime \prime}<\sigma$. At least one $f_{n, \sigma}$ is never zero on some open interval containing $x$, thus $f_{n, \sigma}$ becomes a unit in $R_{m_{x}}^{\prime}$. For all $\sigma^{\prime}>\sigma, f_{n, \sigma^{\prime}}$ is never zero on some open interval containing $x$ so every $f_{n, \sigma^{\prime}}$ becomes a unit in $R_{m_{x}}^{\prime}$ for $\sigma^{\prime}>\sigma$. Thus $\mathcal{Q} R_{m_{x}}^{\prime}$ is generated by $\left\{\bar{Z}_{\sigma^{\prime}}-\bar{Z}_{\sigma^{\prime \prime}}: \sigma^{\prime \prime}>\sigma^{\prime} \geq \sigma\right\}$. Therefore, for all $\sigma^{\prime}$ $>\sigma, \bar{Z}_{\sigma}=\bar{Z}_{\sigma^{\prime}}$ in $R_{m_{x}}$ and the set $\left\{Z_{\sigma^{\prime \prime}}: 0<\sigma^{\prime \prime} \leq \sigma\right\}$ is algebraically independent in $R_{m_{x}}$ over $A_{m_{x}}$, since $R_{m_{x}}^{\prime}=A_{m_{x}}\left[\left\{\bar{Z}_{\sigma^{\prime}}: \sigma \in W\right\}\right], R_{m_{x}}=A_{m_{x}}\left[\left\{Z_{\sigma^{\prime \prime}}: \sigma^{\prime \prime} \leq \sigma\right\}\right]$. If $x=\alpha$, then every $f_{n, \sigma}$ is in the kernel of the canonical map from $R^{\prime}$ to $R_{m \alpha}^{\prime}$ Therefore $\mathcal{Q} R_{m_{\alpha}}^{\prime}=0$ and $R_{m_{\alpha}} \cong R_{m_{a}}^{\prime}$ is also a polynomial ring over $A_{m_{a}}$. Since $R_{m}$ is a polynomial ring over $A_{m}$ for every maximal ideal $m$ of $A, R$ is locally polynomial over $A$.

It is obvious from this example that it is possible for a ring $R$ to be locally a polynomial ring over $\Lambda$, for $R_{p}$ to be a polynomial ring over $\Lambda_{p}$ in only finitely many variables, and yet as one ranges over the primes of $\Lambda$, the number of indeterminates may grow arbitrarily large. This cannot happen for rings with only finitely many minimal prime ideals (for instance, noetherian rings). For if $R$ is locally polynomial over such a ring in a finite number of indeterminates, let $p_{1}, \cdots, p_{n}$ be the height zero primes of $A$. For each $i$, let $m_{i}$ be the number of indeterminates for $R_{p_{i}}$ over $A_{p_{i}}$. Then $\max \left\{m_{i}\right\}$ certainly exists. Since every prime of $\Lambda$ contains a height zero prime, by (1.14), the number of indeterminates for $R_{p}$ over $A_{p}$ is at most $\max \left\{m_{i}\right\}$.

Under some circumstances we are able to classify the locally polynomial rings. If $R$ is an integral domain containing another domain $D$, the transcendence degree of $R$ over $D$ is defined to be the transcendence degree of the quotient field of $R$ over the quotient field of $D$.

(1.19) Let $R$ be a domain of transcendence degree one over a domain $D$ and suppose that $R \subseteq D\left[x_{1}, \ldots, x_{n}\right]$ where the set $\left\{x_{1}, \ldots, x_{n}\right\}$ is algebraically in. dependent over $D$. If $R$ is locally polynomial over $D$, then there is a fractional ideal $\mathcal{U}$ of $D$ and a $t \in R$ such that $R=D[\mathfrak{Q t} t]$.

Proof. If $K$ is the quotient field of $D$ and if $D^{*}=D-\{0\}$, then $R_{D^{*}}=K[t]$ for some $t$ which we may assume is in $R$. We can also assume that as an element of $D\left[X_{1}, \ldots, X_{n}\right], t$ has constant term 0 . Let $p$ be any prime of $D$; then $R_{p}=$ $D_{p}\left[t_{p}\right]$ for some element $t_{p}$ which can also be taken from $R$ with constant term 0 . Then $K[t]=\left(R_{p}\right)_{D^{*}}=K\left[t_{p}\right]$; thus there are elements $a_{p}$ and $b_{p}$ in $K$ such that $t_{p}=a_{p} t+b_{p}$. Since both $t_{p}$ and $t$ have constant term 0 in $D\left[X_{1}, \cdots, X_{n}\right], b_{p}=0$ and $t_{p}=a_{p} t$. Let $\mathcal{Q}=\Sigma_{p \in \text { spec }} D_{p} D$. Then $a$ is a $D$-submodule of $K$ and $D\left[\left\{t t^{n}\right]=\right.$ $D\left[\left\{a_{p} t\right\}\right] \subseteq R$. For any prime $q$ of $D,\left(D\left[\left\{a_{p} t\right\}\right]\right)_{q}=R_{q}$. Thus $D[\{t]]=R$. Let $d$ be any nonzero coefficient of $t$ as an element of $D\left[X_{1}, \ldots, X_{n}\right]$. Then, since $a_{p} t \epsilon$ $R \subseteq D\left[X_{1}, \cdots, X_{n}\right], d a_{p} \in D$ for every $p$. Thus $d \mathscr{U} \subseteq D$ and $\mathscr{U}$ is a fractional ideal of $D$. 
The ideal $\mathscr{O}$ above is locally principal, and $R$ is a polynomial ring over $D$ if and only if $\mathscr{U}$ is a principal ideal; $R$ is an affine domain over $D$ if the ideal $\mathscr{U}$ is finitely generated. The next example shows that the converse to (1.19) is false even if $D$ is assumed to be noetherian and $R$ is an affine ring over $D$.

(1.20) Example. Let $k$ be a field and let $X$ and $Y$ be indeterminates over $k$. Set $D=k[X, Y]_{(X, Y)}$. Let $T$ be an indeterminate over $D$ and set $R=D[X T, Y T]$. Taking $f=X Y T$ and $\mathfrak{U}=(1 / X) D+(1 / Y) D$, we have $R=D[\mathfrak{U} f] \subseteq D[T]$, but since $D$ is local, $R$ is certainly not locally polynomial over $D$.

We do however have the following result.

(1.21) Suppose that $D$ is a noetherian domain and $R$ is of transcendence degree one over $D$ such that $R \subseteq D\left[X_{1}, \cdots, X_{n}\right]$ where $X_{1}, \cdots, X_{n}$ are algebraically independent over $D . R$ is locally polynomial over $D$ if and only if there is an $f \in R$ and an invertible ideal 2 of $D$ such that $R=D[\mathbb{Q} f]$.

Proof. For the necessity, we first observe that there is an $f$ in $R$ and a fractional ideal $\mathscr{U}$ of $D$ such that $R=D[\mathscr{U} f]$. Since $R$ is locally polynomial over $D$ in one variable, the ideal $\mathfrak{O}$ is locally principal and hence locally invertible. Since $D$ is noetherian $\mathcal{U}$ is invertible. Conversely, if the ideal $\mathscr{O}$ is invertible it is locally principal and $R$ is locally polynomial over $D$.

In the event that the ground ring is a quasi-semi-local domain, in the one variable case, the only locally polynomial rings are in fact polynomial rings.

(1.22) Suppose that $D$ is a quasi-semi-local domain. If $R$ is locally polynomial over $D$ in one variable, then $R$ is a polynomial ring over $D$.

Proof. We proceed by induction on the number of maximal ideals, $n$. It is certainly true for $n=1$. Suppose that it is true for $n=k$ and let $D$ be a domain with $k+1$ maximal ideals, $p_{1}, \cdots, p_{k+1}$. Let $M=D-p_{1} \cup \cdots \cup p_{k}$. Then $R_{M}$ is locally polynomial over $D_{M}$ and by the induction hypothes is there is a $t_{1}$ in $R_{M}$ such that $R_{M}=D_{M}\left[t_{1}\right]$. We can in fact assume that $t_{1}$ is in $R . R_{p_{k+1}}=D_{p_{k+1}}\left[t_{2}\right]$ and we can assume that $t_{2}$ is in $R$. Choose $a_{1}$ in $p_{k+1}$ and not in $p_{1} \cup \cdots \cup p_{k}$. Let $a_{2}$ be in $p_{1} \cap \cdots \cap p_{k}$ but not in $p_{k+1}$. Set $t=a_{1} t_{1}+a_{2} t_{2}$ and $R^{\prime}=D[t]$. If $K$ is the quotient field of $D$ then $K\left[t_{1}\right]=K\left[t_{2}\right]$. Thus there are elements $b$ and $c$ in $K$ such that $t_{2}=b+c t_{1}$. Since $t_{2} \in R \subseteq D_{M}\left[t_{1}\right], b, c \in D_{M}$. Let $r_{1}=$ $\left(a_{1}+a_{2} c\right)^{-1}$ and $r_{2}=-a_{2} b r_{1}$. Now $r_{1}$ and $r_{2}$ are in $D_{M}$ and

$$
r_{1} t+r_{2}=\frac{a_{1} t_{1}+a_{2} t_{2}-a_{2} b}{a_{1}+a_{2} c}=\frac{a_{1} t_{1}+a_{2} b+a_{2} c t_{1}-a_{2} b}{a_{1}+a_{2} c}=t_{1} .
$$

Therefore $t_{1} \in D_{M}[t]=R_{M}^{\prime}$. Thus $R_{M}^{\prime}$ contains $R_{M}$. Since the reverse inclusion is obvious, $R_{M}^{\prime}=R_{M}$. Similarly $R_{p_{k+1}}^{\prime}=R_{p_{k+1}}$ and therefore $R=R^{\prime}$. 
(2.0) If $R$ is a polynomial ring over a ring $A$ the content of an element of $R$ over $A$ is defined to be the ideal of $A$ which is generated by the coefficients of that element. In this chapter we examine the extension of this concept to locally polynomial rings. We begin by examining the possibility of extending it to modules.

(2.1) Definition. If $M$ is a module over a ring $R$, then for any $x \in M$ we define the content of $x$ to be the smallest integral ideal $A_{x}(M, R)$ such that $x \in$ $A_{x}(M, R) M$. We call $M$ a content module over $R$ if $A_{x}(M, R)$ exists for every ele. ment $x$ of $M$.

Whenever no confusion will result, we omit the $M$, the $R$, or both from the notation.

(2.2) The module $M$ is a content module over $R$ if and only if for every collection $\left\{\mathscr{U}_{a}\right\}_{a \in \mathbb{Q}}$ of integral ideals of $R, \bigcap_{a \in \mathbb{Q}}\left(\mathscr{U}_{a} M\right)=\left(\bigcap_{a \in \mathbb{Q}} \mathfrak{U}_{a}\right) M$.

Proof. Suppose that $M$ is a content module over $R$ and let $\left\{\mathscr{T}_{\alpha}\right\}_{a \in \mathbb{Q}}$ be a collection of ideals in $R$. Let $x \in \bigcap_{a \in \mathbb{Q}}\left(\mathscr{U}_{a} M\right)$. Then $x \in \mathscr{U}_{a} M$ for all $a$ implies that $A_{x} \subseteq \mathcal{U}_{\alpha}$ for all $\alpha$. Therefore, $A_{x} \subseteq \bigcap_{a \in \mathbb{Q}}\left(\mathscr{U}_{\alpha}\right)$ and $x \in\left(\bigcap_{a \in \mathbb{Q}} \mathscr{U}_{\alpha}\right) M$. Thus $\bigcap_{a \in \mathbb{Q}}\left(\mathscr{A}_{\alpha} M\right) \subseteq\left(\bigcap_{a \in \mathbb{Q}} \mathfrak{U}_{\alpha}\right) M$. Since the reverse inclusion is obvious, we have equality. Conversely, suppose that $x \in M$ and let $\left\{\mathscr{R}_{\alpha}\right\}_{a \in \mathbb{Q}}$ be the collection of all ideals in $R$ such that $x \in \mathscr{U}_{\alpha}$ for each $a \in \mathbb{Q}$. Then $x \in\left(\bigcap_{\alpha \in \mathbb{Q}} \mathscr{P}_{\alpha}\right) M$ and this is certainly the smallest ideal with this property. Thus $A_{x}=\bigcap_{a \in \mathbb{Q}} \mathfrak{U}_{\alpha}$.

(2.3) Remark. If $x \in M$ has content $A_{x}$ over $R$, then there exist elements $a_{1}, \ldots, a_{n}$ in $A_{x}$ and $m_{1}, \ldots, m_{n}$ in $M$ such that $x=\sum_{i=1}^{n} a_{i} m_{i}$. Then $x \in$ $\left(a_{1}, \ldots, a_{n}\right) M$ implies that $A_{x} \subseteq\left(a_{1}, \ldots, a_{n}\right)$. Therefore $\left(a_{1}, \ldots, a_{n}\right)=A_{x}$. Thus content ideals are always finitely generated and the condition $(\bigcap \mathcal{U}) M=\bigcap(\mathcal{U} M)$ for arbitrary families of ideals is equivalent to this condition for arbitrary families of finitely generated ideals.

(2.4) If $M=\bigoplus_{i \in I} M_{i}$ is a module over a ring $R$, then $M$ is a content module if and only if each $M_{i}$ is a content module.

Proof. Suppose $M$ is a content module. For each $i$, there is some $M_{i}^{\prime}$ such that $M=M_{i} \oplus M_{i}^{\prime}$. For any ideal $\mathscr{U}$ of $R$ and for $x \in M_{i}, x \in \mathscr{U M} M_{i}$ if and only if $(x, 0) \in \mathfrak{Q Y M}$. Thus $A_{x}=A_{(x, 0)}$. Conversely, suppose that each $M_{i}$ is a content module. If $\mathfrak{Q}$ is any ideal of $R, \mathfrak{Q}\left(\bigoplus_{I} M_{i}\right)=\bigoplus_{i \in I} \mathfrak{R} M_{i}$. Therefore, if $\{\mathscr{Q}\}_{\alpha \in \mathbb{Q}}$ is any collection of ideals of $R$, we have

$$
\begin{aligned}
\bigcap_{a \in \mathbb{Q}}\left(\mathfrak{U}_{a} \bigoplus_{i \in I} M_{i}\right) & =\bigcap_{a \in \mathbb{Q}}\left(\bigoplus_{i \in I}\left(\mathscr{U}_{\alpha} M_{i}\right)\right)=\bigoplus_{i \in I}\left(\bigcap_{a \in \mathbb{Q}}\left(\mathfrak{U}_{a} M_{i}\right)\right) \\
& =\bigoplus_{i \in I}\left(\left(\bigcap_{a \in \mathbb{Q}} \mathfrak{U}_{a}\right)^{M_{i}}\right)=\left(\bigcap_{a \in \mathbb{Q}} \mathfrak{U}_{a}\right)\left(\bigoplus_{i \in I}^{\bigoplus_{i}}\right)
\end{aligned}
$$

Thus $M$ is a content module by (2.2). 
A free module over a ring $R$ is a direct sum of copies of $R$. Since $R$ is obviously a content module over itself, every free module is a content module. Every projective module is a direct summand of a free module. Thus we have the following as a corollary to (2.4).

(2.5) Every projective module over a ring $R$ is a content module over $R$.

If $R$ is a noetherian ring, we have a similar result for direct products.

(2.6) Suppose that $R$ is a noetberian ring. If $M=\Pi_{i \in I} M_{i}$ is a product of $R$ modules, then $M$ is a content module if and only if each $M_{i}$ is a content module.

Proof. If $M$ is a content module, then each $M_{i}$ is a direct summand of a content module and is therefore a content module by (2.4). Suppose that each $M_{i}$ is a content module. Since $R$ is noetherian, any ideal $a$ of $R$ is finitely generated and $\mathcal{Q} \Pi_{i \in I} M_{i}=\Pi_{i \in I} \mathscr{Q} M_{i}$. Thus, if $\left\{\mathscr{Q}_{a}\right\}_{a \in \mathbb{Q}}$ is any collection of ideals of $R$, we have

$$
\begin{aligned}
\bigcap_{a \in \mathbb{Q}}\left(\mathfrak{U}_{a} \prod_{i=I} M_{i}\right) & =\bigcap_{a \in \mathbb{Q}}\left(\prod_{i \in I}\left(\mathscr{U}_{a} M_{i}\right)\right)=\prod_{i \in I}\left(\bigcap_{a \in \mathbb{Q}}\left(\mathscr{U}_{a} M_{i}\right)\right) \\
& =\prod_{i \in I}\left(\left(\bigcap_{a \in \mathbb{Q}} \mathscr{U}_{a}\right) M_{i}\right)=\left(\bigcap_{a \in \mathbb{Q}} \mathscr{U}_{a}\right) \prod_{i \in I} M_{i}
\end{aligned}
$$

Thus $M$ is a content module by (2.2).

(2.7) Suppose that $M$ is a content module over a ring $R$. If $\mathscr{N}$ is an ideal of $R$, then $M / \mathscr{Q} M$ is a content module over $R / \mathscr{U}=R^{\prime}$ and for any $x \in M, \overline{A_{x} R}$ $A_{\bar{x}}\left(R^{\prime}\right)$.

Proof. If $x \in A_{x}(R) M$ then $\bar{x} \in \overline{A_{x}(R) M}=\overline{\left(A_{x}(R)\right)(M)}$. Suppose that $I$ is an ideal of $R^{\prime}$ such that $\bar{x} \in I \bar{M}$. There is an ideal 9 of $R$ which contains $\mathscr{Q}$ such

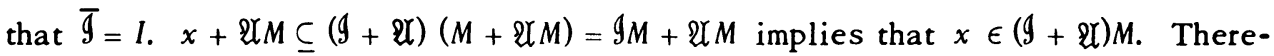
fore, $A_{x}(R) \subseteq 9+\mathfrak{Q}=9$ and $\overline{A_{x}(R)} \subseteq 1$. Thus $\overline{A_{x}(R)}=A_{\bar{x}}\left(R^{\prime}\right)$ and $M / \mathfrak{Q} M$ is a content module.

(2.8) Suppose that $f: R \rightarrow R^{\prime}$ makes $R^{\prime}$ an $R$-algebra. If $R^{\prime}$ is a content module over $R$ and if $M$ is a content module over $R^{\prime}$, then $M$ is a content module over $R$.

Proof. If $\left\{\mathcal{A}_{\alpha}\right\}_{a \in Q}$ is any collection of ideals in $R$, then $\left\{f\left(\mathscr{U}_{\alpha}\right) R^{\prime}\right\}_{a \in Q}$ is a collection of ideals of $R^{\prime}$. Thus

$$
\bigcap_{a \in \mathbb{Q}} f\left(\mathscr{U}_{\alpha}\right) M=\bigcap_{a \in \mathbb{Q}} f\left(\mathscr{U}_{\alpha}\right) R^{\prime} M=\left(\bigcap_{a \in \mathbb{Q}} f\left(\mathscr{U}_{\alpha}\right) R^{\prime}\right) M=\left(\bigcap_{a \in \mathbb{Q}} f\left(\mathscr{U}_{\alpha}\right)\right)^{M}
$$

By (2.2), $M$ is a content module over $R$.

If the $R$-algebra is a quotient ring of $R$, then flat content modules become content modules over $R^{\prime}$. 
(2.9) Suppose that $M$ is a flat content module over a ring $R$ and $S$ is a multiplicative system in $R$. Then $M_{S}$ is a content module over $R_{S}$ and for every element of the form $m \otimes 1 / s$ in $M_{S}, A_{m \otimes 1 / s}\left(R_{S}\right)=A_{m}(R) R_{S}$.

Proof. Let $\mathfrak{b}$ be the ideal consisting of annihilators of elements of $S$. By (2.7) and by the fact that $M / \mathscr{G} M \cong M \otimes R / \mathcal{G}$ is flat over $R / \mathcal{G}$ we can assume that $S$ is a regular multiplicative system. Note that all elements of $M_{S}$ are of the form $m \otimes 1 / s$. Suppose that, for some ideal $\mathcal{U}$ of $R_{S}, m \otimes 1 / s \in M_{S}$. Then there are elements $a_{1}, \cdots, a_{n}$ in $R$ and elements $m_{1} \otimes 1 / s_{1}, \ldots, m_{n} \otimes 1 / s_{n}$ in $M_{s}$ such that $m \otimes 1 / s=\sum_{i=1}^{n} a_{i}\left(m_{i} \otimes 1 / s_{i}\right)$. Multiplying both sides by a suitable element of $S$ we have $s^{\prime} m \otimes 1=\sum_{i=1}^{n} a_{i}\left(s_{i}^{\prime} m_{i} \otimes 1\right)=\left(\sum_{i=1}^{n} a_{i} s_{i}^{\prime} m_{i}\right) \otimes 1$. Therefore $\left(s^{\prime} m-\right.$ $\left.\sum_{i=1}^{n} a_{i} a_{i}^{\prime} m_{i}\right) \otimes 1=0$. Thus we have $s^{\prime} s^{\prime \prime} m-\sum_{i=1}^{n} a_{i} s^{\prime \prime} s_{i}^{\prime} m_{i}=0$ for some $s^{\prime \prime}$ in $S$. Let $\mathcal{S}$ be the set of all regular elements of $R$ and consider the exact sequence 0 $\rightarrow R \rightarrow R_{\mathcal{S}}$. Since $M$ is flat, $0 \rightarrow M \otimes R \rightarrow M \otimes R_{\mathcal{S}}$ is exact and $M$ can be idenfied with the submodule $M \otimes R$ of $M \otimes R_{\boldsymbol{\delta}}$. As such, $a M=M \otimes \mathscr{Q}$ is well defined and we have $s^{\prime} s^{\prime \prime} m \in \mathfrak{U} M$. Thus $m \in M \otimes \mathfrak{U} / s^{\prime} s^{\prime \prime}$. Therefore $m \in\left(M \otimes \mathfrak{U} / s^{\prime} s^{\prime \prime}\right) \cap$ $(M \otimes R)=M \otimes\left(\mathfrak{U} / s^{\prime} s^{\prime \prime} \cap R\right)=M \otimes \mathscr{U}^{c}=\mathfrak{U}^{c} M$. Then $A_{m}(R) \subseteq \mathfrak{U}^{c}$ and $A_{m}(R) R_{s}$ $\subseteq \mathcal{Q}$. Since it is clear that $m \otimes 1 / s \in A_{m}(R) M_{S}$, we see that $A_{m}(R) R_{S}=A_{m \otimes 1 / s}\left(R_{S}\right)$.

This gives us the following corollary.

(2.10) If $M$ is a flat content module over a ring $R$ and $s$ is a regular element of $R$, then for every element $x$ of $M, A_{s x}=s A_{x}$.

Proof. Since $s x \in s A_{x} M$, we see that $A_{s x} \subseteq s A_{x}$. Let $S$ be the multiplicative system of $R$ generated by $s$. The sequence $0 \rightarrow R \rightarrow R_{S}$ is exact since $s$ is regular. Since $M$ is flat, the sequence $0 \rightarrow M \otimes R \rightarrow M \otimes R_{S}$ is also exact. Thus $M$ can be considered as a submodule of $M_{S}$. Since $s x \in A_{s x} M, x \in\left(A_{s x} / s\right) M \cap M$ $=\left(A_{s x} / s \cap R\right) M$. Thus $A_{x} \subseteq\left(A_{s x} / s \cap R\right)$. Therefore, $s A_{x} \subseteq s\left(A_{s x} / s \cap R\right)=A_{s x}$ $\cap s R \subseteq A_{s x}$ and $s A_{x}=A_{s x}$.

Another application of (2.9) gives the following result.

(2.11) Suppose that $M$ is a flat content module over a ring $R$ and that $x \in M$. The module $M^{\prime}=M /(x)$ is flat over $R$ if and only if $A_{x}$ is generated by an idem. potent.

Proof. Suppose that $M^{\prime}$ is flat over $R$. Let $p$ be any prime of $R$ and consider the exact sequence

$$
0 \rightarrow(x)_{p} \rightarrow M_{p} \rightarrow M_{p}^{\prime} \rightarrow 0 .
$$

Let $\bar{x}$ be the image of $x$ in $M_{p}$. Since both $M$ and $M^{\prime}$ are content and flat, $M_{p}$ and $M_{p}^{\prime}$ are both free over $R_{p}$. Thus $\left(x_{p}\right.$ is either zero or is a direct summand of $M_{p}$. If $(x)_{p}=(0)$, then $x=0$ and $A_{\bar{x}}\left(R_{p}\right)=(0)$. Otherwise, since $\bar{x} \in A_{\bar{x}}\left(R_{p}\right) M_{p}$, 
we must have $\bar{x} \in A_{\bar{x}}\left(R_{p}\right)(x)_{p}$. Therefore, $A_{\bar{x}}\left(R_{p}\right)(x)_{p}=(x)_{p}$ and $\bar{x} \in\left(A_{\bar{x}}\left(R_{p}\right)\right)^{2}(x)_{p}$. Since $A_{\bar{x}}\left(R_{p}\right)$ is minimal with respect to this property, $\left(A_{\bar{x}}\left(R_{p}\right)\right)^{2}=A_{\bar{x}}\left(R_{p}\right)$. By (2.9), for every prime $p$ of $R, A_{x} R_{p}=\left(A_{x}\right)^{2} R_{p}$. Therefore, $A_{x}$ is an idempotent ideal of $R$. Since it is also finitely generated, it is generated by an idempotent. Conversely, suppose that $A_{x}$ is generated by an idempotent. Then for every prime $p$ of $R, A_{x} R_{p}$ is either (0) or $R_{p}$. If it is $(0)$, then $(x)_{p}=(0)$ and $M_{p}^{\prime}=M_{p}$. Thus $M_{p}^{\prime}$ is flat. If $A_{x} R_{p}=A_{\bar{x}}\left(R_{p}\right)=R_{p}$, let $\left\{X_{i}\right\}_{i \in l}$ be a free basis for $M_{p}$. Then $\bar{x}=$ $\sum_{i=1}^{n} a_{i} x_{i}$ and $\left(a_{1}, \cdots, a_{n}\right)=R_{p}$. Since $R_{p}$ is quasi-local, some $a_{i}$ must be a unit. Suppose that it is $a_{1}$. Then $x=\left(a_{1}^{-1}\right) \bar{x}-\sum_{i=2}^{n} a_{1}^{-1} a_{i} x_{i}$. Therefore, $x_{1}, x_{2}, \cdots, x_{n}$ is a basis for $M_{p}$ and $M_{p}^{\prime}$ is free. Thus, for any $p, M_{p}^{\prime}$ is $R_{p}$-flat and consequently $M^{\prime}$ is $R$-flat.

In (2.5) we noted that every projective module is a content module. The following example shows that the converse is not in general true.

(2.12) Example. Let $R$ be a noetherian ring which has a proper, infinitely descending chain of ideals. Then by [Ch, Theorem (3.4)], there is an indexing set $I$ which depends upon $R$ and such that the module $M=I_{i \in I} R_{i}$, where each $R_{i}$ is isomorphic to $R$, is not projective. By (2.6) $M$ is a content module over $R$.

We note that the module in example (2.12) is torsion free, but not finitely generated. There are simple examples of finitely generated content modules which are not projective.

(2.13) Example. Let $V$ be a valuation ring, 2 a nonzero ideal of $V$ and $M=$ $V /$ Ul. Then $M$ is a finitely generated content module over $V$ which is not projective.

That $V / \mathscr{Q}$ is a content module is easy to verify and it could not be projective since it is not torsion free.

(2.14) Suppose that $R$ is a domain and $M$ is a finitely generated content module over $R$. The following are equivalent:

(i) $M$ is flat,

(ii) $M$ is projective,

(iii) $M$ is torsion free.

Proof. Since $M$ is a finitely generated module over a domain, (i) implies (ii) by [V, Theorem 1.4]. If $M$ is projective, then it is a direct summand of a free module. Thus it must be torsion free and we have that (ii) implies (iii). Since $M$ is a content module, for any two ideals $\mathscr{A}$ and $\mathfrak{b}$ of $R$, $\mathfrak{Q} M \cap \mathfrak{b} M=(\mathfrak{Q} \cap \mathfrak{b}) M$ by (2.2). Thus $M$ is flat by [Je, Theorem 1] and (iii) implies (i).

(2.15) Suppose that $M$ is a content module over a ring $R$ and that $x \in M$. If

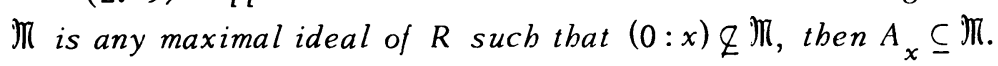

Proof. If $(0: x) q M$, then there is an $a \in(0: x)$ and a $b \in \mathbb{M}$ such that $a+b$ 
=1. $x=x(a+b)=a x+b x=b x$ is in MM. Thus $A_{x} \subseteq M$.

For a ring $R$ let Spec $R$ represent the set of maximal ideals of $R$.

(2.16) Let $D$ be a domain such that, for all $d \in D, \bigcap_{\Re \in \operatorname{spec} D ; d \notin M}=0$. If $M$ is a content module over $D$, then $M$ is torsion free.

Proof. Suppose that for some $x \in M$, there is an $a \in D$ such that $a x=0$. By (2.15), $A_{x}$ is contained in every maximal ideal which does not contain $a$. Thus, $A_{x}=0$ and $x=0$.

As an immediate corollary to (2.12) we have

(2.17) If $D$ is a Hilbert domain, then every content module over $D$ is torsion free and every finitely generated content module is projective.

Proof. Any Hilbert domain satisfies (2.12).

(2.18) If $M$ is a module over a ring $R$ and $A$ is a submodule of $M$, then $M / A$ is a content module if and only if for every collection $\left\{\hat{q}_{\alpha}\right\}_{a \in \mathbb{Q}}$ of ideals of $R$

$$
\bigcap_{a \in \mathbb{Q}}\left(\mathfrak{U}_{\alpha} M+A\right)=\left(\bigcap_{a \in \mathfrak{Q}} \mathfrak{U}_{a}\right) M+A \text {. }
$$

Proof. Let $f: M \rightarrow M / A$ be the canonical homomorphism. For any ideal $\mathcal{O}$ of $R, \mathscr{U}(M / A)=f(\mathscr{U} M+A)$. If $\left\{\mathscr{Q}_{a}\right\}_{a \in \mathbb{Q}}$ is any collection of ideals of $R$, then $\bigcap_{a \in \mathbb{Q}}\left(\mathcal{U}_{\alpha}(M / A)\right)=\bigcap_{a \in \mathbb{Q}} f\left(\mathscr{U}_{a} M+A\right)$. Since the kernel, $A$, is contained in each $\mathcal{U}_{\alpha} M+A$, we have $\bigcap_{a \in \mathbb{Q}}\left(\mathcal{U}_{\alpha}(M / A)\right)=f\left(\bigcap_{\alpha \in \mathbb{Q}}\left(\mathcal{U}_{a} M+A\right)\right)$. In addition, $\left.\left(\bigcap_{a \in \mathbb{Q}} \mathfrak{U} \alpha\right)(M / A)=f\left(\left(\bigcap_{a \in \mathbb{Q}} \mathfrak{U}\right)_{\alpha}\right) M+A\right)$. Since both $\bigcap_{\alpha \in \mathbb{Q}}\left(\mathfrak{U}_{\alpha} M+A\right)$ and $\left(\bigcap_{\alpha \in \mathbb{Q}} \mathfrak{U}{ }_{\alpha}\right) M$ $+A$ contain $A, \bigcap_{a \in \dot{Q}}\left(\mathscr{U}_{\alpha}(M / A)\right)=\left(\bigcap_{a \in \mathbb{Q}^{\mathfrak{R}}}\right)(M / A)$ if and only if $\bigcap_{a \in \mathbb{Q}}\left(\mathscr{U}_{\alpha} M+A\right)$ $=\left(\bigcap_{a \in \mathbb{Q}} \mathfrak{P r}_{\alpha}\right) M+A$.

Over a Hilbert domain, we can characterize these homomorphisms in terms of a more accessible condition.

(2.19) Let $D$ be a Hilbert domain. If $M$ is a finitely generated content module over $D$ with submodule $A$, then $M / A$ is a content module if and only if $A$ is a direct summand of $M$.

Proof. If $M / A$ is a content module, then, since it is finitely generated, it is projective by (2.17). Therefore the sequence $0 \rightarrow A \rightarrow M \rightarrow M / A \rightarrow 0$ splits and $A$ is therefore a direct summand of $M$. Conversely, if $A$ is a direct summand of $M$, then $M$ is $D$-isomorphic to $A \oplus M / A$. Hence $M / A$ is $D$-isomorphic to a direct summand of $M$ and is therefore a content module over $D$ by (2.4).

We can see that it is not in general true that a homomorphic image of a content module is a content module. For example, the Hilbert domain $Z$ when considered as a module over itself is a content module by (2.5). Its submodule $2 Z$ is not a direct summand, and therefore by (2.19) $Z / 2 Z$ is not a content module. 
If $R$ is locally polynomial over a ring $A$, we define the content of any element in $R_{S}$ where $S$ is the set of all regular elements of $A$.

(2.20) Definition. Suppose that $R$ is locally polynomial over $A$ and let $S$ be the set of all regular elements of $A$. For $f$ in $R_{S}$, we define the content of $f$ to be the smallest fractional ideal $A_{f}$ of $A$ such that $f \in A_{f}$.

If an element $f$ of $R$ has content in the sense of (2.20), then $f \in A_{f} R \cap A R$. Since $R$ is faithfully flat over $A$ by $(1.2)$, this ideal is $\left(A_{f} \cap A\right) R$. Since $A_{f}$ is minimal by definition, we must have $A_{f} \subseteq A$. Thus for elements in $R$, this agrees with our previous definition.

(2.21) Suppose that $R$ is locally polynomial over a ring $A$ and let $S$ be the set of all regular elements of $A$, then $A_{f}$ exists for every element $f \in R_{S}$ if and only if $R$ is a content module over $A$.

Proof. Suppose that $R$ is a content module over $A$ and let $f \in R_{S}$. Let $f=$ $f^{\prime} / s$ for some $f^{\prime} \in R$ and some regular element $s$ of $A^{\prime}$ then $A_{f}{ }^{\prime}$ exists and since $f^{\prime} \in A_{f}, R$, we have $f \in 1 / s A_{f}, R$. If $\mathscr{O}$ is any fractional ideal of $A$ such that $f \epsilon$ $\mathfrak{U} R$, then $f^{\prime} \in s \mathfrak{U} R$. Therefore $f \in \mathfrak{U} R \cap A R=(\mathfrak{U} \cap A) R$. Thus $A_{f^{\prime}} \subseteq s \mathfrak{U}$ and $1 / s A_{f^{\prime}}$ $\subseteq \mathfrak{A}$. Hence $A_{f}=1 / s A_{f}$. Since the converse is clear, this completes the proof.

In the event that $R$ is actually a polynomial ring over $A$, then this definition agrees with the usual definition of content. We consider the possibility of generalizing the usual theorems on the content of polynomial rings to locally polynomial rings.

(2.22) If $R$ is locally polynomial over a ring $A$ and is furthermore a content module over $A$, then for any $f \in R_{S}$, where $S$ is the set of regular elements of $A$, $f \in R$ if and only if $A, \subseteq A$.

(2.23) Let $S$ be the set of all regular elements of a ring $A$. If $R$ is locally polynomial over $A$ and is a content module over $A$, then for elements $f, g \in R_{S}$ and $\alpha \in A_{s}$ we bave $A_{f+g} \subseteq A_{f}+A_{g}, A_{f g} \subseteq A_{f} A_{g}$ and $A_{\text {af }}=\alpha A_{f}$. If $\beta$ is a regu. lar element of $A_{S}$ then $A_{\beta f}=\beta A_{f}$.

Proof. Clearly $f+g \in A_{f} R+A_{g} R=\left(A_{f}+A_{g}\right) R$, hence $A_{f+g} \subseteq A_{f}+A_{g}$. Similarly $A_{f g} \subseteq A_{f} A_{g}$ since $f g \in\left(A_{f} R\right)\left(A_{g} R\right)$. Since $a f \in \alpha A_{f} R, A_{a f} \subseteq \alpha A_{f}$. If $\beta$ is a regular element of $\beta A_{f} \subseteq A_{\beta f}$, so $\beta A_{f}=A_{\beta f}$.

Suppose that $R$ is locally polynomial over a ring $A$ and that $S$ is the set of regular elements of $A$. If $p$ is any prime of $A$, let $\phi: A \rightarrow A_{p}$ be the usual map. Every element of $\phi(S)$ is regular in $A_{p}$. Thus by the permutability of residue class and quotient ring formation, there is a map $\phi^{\prime}$ making the following diagram commutative. 


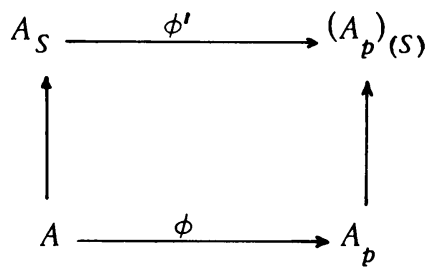

Furthermore, the vertical maps are injective. Suppose that $T_{p}$ is a total quotient ring of $A_{p}$. Tensoring with $R$, we have the following commutative diagram.

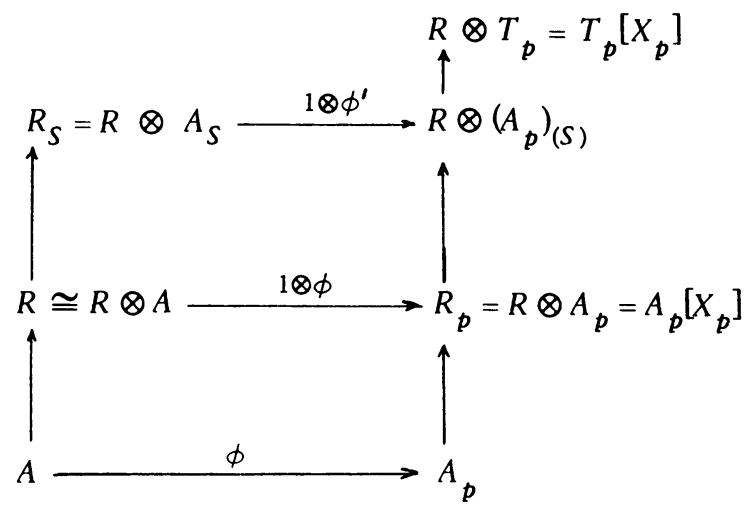

Here $X_{p}$ is an algebraically independent set over $A_{p}$. Since $R$ is flat over $A$, all the vertical maps are injections. Suppose that $R$ is a content module over $A$. Then for any $f \in R_{S}$ the content of $f$ over $A$ is well defined. We denote this by $A$ as usual. Now $f$ is of the form $f^{\prime} \otimes(1 / s)$ for some $f^{\prime}$ in $R$ and some $s$ in $S$, and $\left(1 \otimes \phi^{\prime}\right)(f)=f^{\prime} \otimes(1 / \phi(s))$ can be considered an element of $T_{p}\left[X_{p}\right]$. As such it has content over $A_{p}$ which we denote by $A_{f \otimes 1}$.

(2.24) With the notation above, $\phi^{\prime}\left(A_{f}\right) A_{p}=A_{f \otimes 1}$.

Proof. By (2.9) $\phi\left(A_{f^{\prime}}\right) A_{p}=A_{f^{\prime} \otimes 1}$. Thus by (2.23)

$$
\begin{aligned}
\phi^{\prime}\left(A_{f}\right) A_{p} & =\phi^{\prime}\left(1 / s A_{f^{\prime}}\right) A_{p}=1 / \phi(s) \phi\left(A_{f},\right) A_{p}=1 / \phi(s) A_{f^{\prime} \otimes 1} \\
& =A_{1 / \phi(s)\left(f^{\prime} \otimes 1\right)}=A_{f^{\prime} \otimes(1 / \phi(s))}=A_{f \otimes 1} .
\end{aligned}
$$

(2.25) Suppose that $D$ is a domain and $R$ is locally polynomial over $D$. If $R$ is a content module over $D$ and if for any $f_{,} g \in R_{D-\{0\}}, A_{f} A_{g}=A_{f g^{\prime}}$ then $D$ is Prüfer.

Proof. For any maximal ideal $m$ of $D$, let $A_{b}\left(D_{m}\right)$ be the content of any element $b$ of $R_{D-\{0\}}$ over $D_{m}$. Then $A_{f g}\left(D_{m}\right)=A_{f g} D_{m}$ by (2.24) and similarly $A_{f} A_{g} D_{m}=A_{f}\left(D_{m}\right) A_{g}\left(D_{m}\right)$. Thus $A_{f g}\left(D_{m}\right)=A_{f}\left(D_{m}\right) A_{g}\left(D_{m}\right)$. Since $R_{D-\{0\}}$ is the polynomial ring $R_{m}$ localized at the multiplicative system of nonzero elements of $D_{m}, D_{m}$ is Prüfer by $[G,(24.6)]$. Thus $D_{m}$ is a valuation ring and $D$ is Prüfer. 
One of the well-known results on Krull domains is that a polynomial ring in any number of indeterminates over a Krull domain is again a Krull domain. We are interested in determining to what extent this result can be generalized to locally polynomial rings.

(2.26) If $R$ is locally polynomial over a Krull ring $D$, then $R=\bigcap_{p \in \operatorname{spec} D ; \mathrm{ht}(p)=1} R_{p}$.

Proof. For any prime $p$ of $D$, let $\left\{X_{p}\right\}$ be the set of indeterminates which generates $R_{p}$ over $D_{p}$. If $q \subset p$ are primes in $D$, then $R_{q}=D_{q}\left[\left\{X_{q}\right\}\right]$ is a localization of $R_{p}=D_{p}\left[\left\{X_{p}\right\}\right]$ at a multiplicative system which is contained in $D_{p}$. Thus $R_{q}=D_{q}\left[\left\{X_{p}\right\}\right]$. For any $p, D_{p}=\bigcap_{q \subseteq p ; \mathrm{ht}(q)=1} D_{q}$. Therefore

$$
\begin{aligned}
R_{p} & =D_{p}\left[\left\{X_{p}\right\}\right]=\bigcap_{q \subseteq p ; \mathrm{ht}(q)=1} D_{q}\left[\left\{X_{p}\right\}\right] \\
& =\bigcap_{q \subseteq q ; \mathrm{ht}(q)=1} D_{q}\left[\left\{X_{q}\right\}\right]=\bigcap_{q \subseteq p ; \mathrm{ht}(q)=1} R_{q} .
\end{aligned}
$$

By (1.10), we have

$$
R=\bigcap_{p \in \operatorname{spec} D} R_{p}=\bigcap_{q \in \operatorname{spec} D ; \mathrm{ht}(q)=1} R_{q} .
$$

(2.27) If $A$ is a Krull domain and $R$ is locally polynomial over $A$, then the following are equivalent:

(i) $R$ is Krull;

(ii) if $\left\{\mathfrak{U}_{a}\right\}_{a \in \mathbb{Q}}$ is a family of divisorial ideals in $A$, then $\left(\bigcap_{a \in \mathbb{Q}} \mathfrak{Q} \mathbb{Q}_{a} R\right)=$ $\left(\bigcap_{a \in \mathbb{Q}} \mathfrak{H}_{a}\right) R$;

(iii) each nonzero element of $R$ is contained in only finitely many ideals of the form $p R$, for $p$ a beight one prime of $A$.

Proof. (i) implies (ii). $R$ is faithfully flat over $A$ by (1.2). Therefore, $\mathfrak{U}_{\alpha} R$ is a divisorial ideal of $R$ for each $\mathfrak{U}_{a}$. Thus if there is an infinite number of dis. tinct $\mathfrak{U}_{\alpha}^{\prime}$ 's, then $\bigcap_{a \in \mathbb{Q}}\left(\mathfrak{H}_{a} R\right)=0$. Otherwise $\bigcap_{a \in \mathbb{Q}}\left(\mathfrak{A}_{a} R\right)=\left(\bigcap_{a \in \mathbb{Q}} \mathfrak{U}{ }_{a}\right) R$.

(ii) implies (iii). Given any infinite collection $\left\{p_{a}\right\}_{\alpha \in \mathbb{Q}}$ of height one primes of $A, \bigcap_{\alpha \in \mathbb{Q}}\left(p_{\alpha} R\right)=\left(\bigcap_{\alpha \in \mathbb{Q}} p_{\alpha}\right) R=0$. Thus any nonzero element of $R$ can be contained in at most a finite number of the $p R$ 's.

(iii) implies (i). By (2.26), $R=\bigcap_{p \in \operatorname{spec} A ; \mathrm{ht}(p)=1} R_{p}$. Since $R$ is locally polynomial over $A$, for each $p$, there is a set of indeterminates over $A$ which we denote by $X_{p}$ such that $R_{p}=A_{p}\left[X_{p}\right]$. Furthermore, we can assume that $X_{p}$ is contained in $R$. If $A^{*}$ is the set of nonzero elements of $A$, then there is another set of indeterminates $X_{0}$ in $R$ such that $R_{A^{*}}=K\left[X_{0}\right]$ for each prime $p \cdot A_{p}\left(X_{p}\right)$ $\cap K\left[X_{0}\right]=A_{p}\left[X_{p}\right]$. Therefore, $R=\bigcap_{p \in \operatorname{spec} A ; \mathrm{ht}(p)=1} A_{p}\left(X_{p}\right) \cap K\left[X_{0}\right]$. Since $A_{p}\left(X_{p}\right)=R_{(p R)}$ and since every nonzero element of $R$ is contained in at most finitely many of the ideals $p R$, every nonzero element of $R$ is a nonunit in at 
most finitely many of the $A_{p}\left(X_{p}\right)$. Thus $R$ is Krull [S, Proposition 4.1].

As immediate corollaries to (2.27) we have

(2.28) If $R$ is locally polynomial over a Dedekind domain $A$, then $R$ is Krull if and only if $R$ bas content over $A$.

(2.29) If $R$ is locally polynomial over a Krull ring $A$ and $R$ is a content module over $A$, then $R$ is Krull.

(2.30) Remark. In closing the section on content we note that the following question arises naturally:

For which ring $R$ are finitely generated, flat content modules projective?

(3.0) In this section we investigate the stability of certain finiteness conditions under the formation of locally polynomial rings. We begin with a consideration of Krull rings. We refer the reader to [S] for an elegant introduction to Krull rings. Following [S], if $A$ is a Krull ring we let $D(A)$ denote the group of divisors of $A, F(A)$ the group of principal divisors, and $C(A)=D(A) / F(A)$ the divisor class group of $A$. By a straight forward adaptation of the argument given there for polynomial rings we see the following:

(3.1) If $R$ is locally polynomial over a domain $A$ and if $R$ is Krull then $C(A)=C(R)$.

Proof. Since $A=R \cap$ (quotient field $A$ ), $A$ is also Krull. For a divisorial ideal $\mathfrak{U}$ of $A$, define $j(\mathfrak{U})=\mathfrak{U} R$. Since $R$ is flat over $A, j(\mathfrak{U})$ is a divisorial ideal in $R$ and by [S, Theorem 6.1 and Theorem 6.2] this defines a homomorphism which we again denote by $j$ from $D(A)$ to $D(R)$. This induces a homomophism

$$
\bar{j}: C(A) \rightarrow C(R) \text {. }
$$

Since $R$ is faithfully flat over $A$, if $j(\mathfrak{Z})=j(\mathfrak{b})$, then

$$
\mathfrak{U}=\mathfrak{Q} R \cap A=\mathfrak{b} R \cap A=\mathfrak{b}
$$

[B, p. 50], thus $j$ is monic. Moreover $\bar{j}$ is also monic, for suppose that $\mathfrak{U}^{\prime}$ is a divisorial ideal of $A$ such that $j\left(\mathfrak{U}^{\prime}\right)$ is principal. Then there is an $a$ in $A$ such that $a \mathfrak{U}^{\prime}=\mathfrak{U} \subset A$. Now $j(\mathfrak{U})=j\left(a \mathfrak{U} \mathfrak{U}^{\prime}\right)=a j\left(\mathfrak{U}^{\prime}\right)$ is principal. Thus $j(\mathfrak{U})$ is principal. Suppose $\mathscr{U} R=x R$. If $K$ denotes the quotient field of $A$ and $z \in \mathfrak{U}-\{0\}$, then $z R \subseteq x R$ implies $z R_{A^{*}} \subseteq x R_{A^{*}}$ where $A^{*}=A-\{0\}$. Since $R_{A^{*}}$ is a polynomial ring over $K$ there is a well-defined degree. $\operatorname{deg}(x) \leq \operatorname{deg}(z)=0$ and $x \in R \cap K=A$. Since $R$ is faithfully flat over $A, \mathfrak{U} R=x R$ implies that $\mathfrak{U}=x A$. Thus $\bar{j}$ is monic. Set $R_{A^{*}}=K\left[\left\{x_{0}\right\}\right]$ where $\left\{x_{0}\right\}$ is some set of indeterminates over $K$. By [S, Theorem 6.3] we have a homomorphism

$$
j_{1}: D(R) \rightarrow D\left(K\left[\left\{X_{0}\right\}\right]\right)
$$


which induces a homomorphism

$$
\bar{j}_{1}: C(R) \rightarrow C\left(K\left[\left\{x_{0}\right\}\right]\right)
$$

with

$$
\operatorname{ker} \overline{\left(j_{1}\right)}=(H+F(R)) / F(R)
$$

where $H$ is the subgroup of $D(R)$ generated by divisors of height one primes $p$ of $R$ such that $p \cap A \neq(0)$. Since $C\left(K\left[\left\{X_{0}\right\}\right]\right)=0$,

$$
C(R)=(H+F(R)) / F(R) \text { and } D(R)=H+F(R) .
$$

If $p$ is a height one prime of $R$ such that $p \cap A=\mathcal{Y} \neq(0)$, then by (1.11) $\mathcal{Y}$ is a height one prime of $A$ and $j(\mathcal{Y})=p$. Thus $j(D(A))=H$ and $\bar{j}$ is surjective. Therefore, $\bar{j}$ is an isomorphism from $C(A)$ to $C(R)$.

(3.2) Let $A$ be a Krull ring and $R^{\prime}=A\left[\xi_{1}, \ldots, \xi_{n}\right]$ an affine domain over $A$. Suppose $R$ is locally a polynomial ring over $A$ and $A \subseteq R \subseteq R^{\prime}$. Then there are elements $\lambda_{1}, \cdots, \lambda_{e}$ in the quotient field of $R$ such that $A \subseteq R \subseteq A\left[\lambda_{1}, \cdots, \lambda_{e}\right]$.

Proof. We first reduce to the case where $R^{\prime}$ is algebraic over $R$. Let $L$ denote the quotient field of $R$ and $L^{*}$ the nonzero elements of $L$. Then $R^{\prime}\left[L^{*}\right]$ is an affine ring over $L$ and its dimension is the transcendence degree of $R^{\prime}$ over $R[\mathbf{N}, \mathbf{l r}, \mathrm{p}, 46]$. Let $P$ be a prime ideal of $R^{\prime}$ such that $P R^{\prime}\left[L^{*}\right]$ is a maximal ideal of $R^{\prime}\left[L^{*}\right]$. Then $R^{\prime} / P$ will be algebraic over $L[\mathbf{N}, \mathbf{l r}, \mathrm{p} .46]$. Thus if we pass to the situation $A \subseteq R \subseteq R^{\prime} / P$ we may assume that the $\xi_{i}$ are algebraic over $R$. Let $R_{(0)}=K[T]$ where $K$ is the quotient field of $A$ and $T$ is a finite set of elements in $R$ which are algebraically independent over $K$. Since $R^{\prime}$ is affine over $A[T]$ by $[\mathbf{N}, \mathbf{l r},(14.4)]$ there is an $f \in A[T]$ such that $R^{\prime}[1 / f]$ is integral over $A[T, 1 / f]$. We have $A[T, 1 / f] \subseteq R[1 / f] \subseteq R^{\prime}[1 / f]$ and $R^{\prime}[1 / f]$ is integral over $A[T, 1 / f]$. Therefore $R[1 / f]$ is integral over $A[T, 1 / f]$ and since the latter is integrally closed $A[T, 1 / f]=R[1 / f]$. Thus $R \subseteq A[T, 1 / f]$ and we are done.

(3.3) Suppose that $A$ is a Krull ring and $R$ is locally a polynomial ring over A. If there is an affine domain $R^{\prime}$ over $A$ such that $A \subseteq R \subseteq R^{\prime}$ then $R$ is Krull. Moreover, $R$ is an affine ring over $A$.

Proof. By the previous result we may assume that $R^{\prime}$ has the same quotient field as $R$. Fix a prime $p$ and let $X_{p}$ denote a set of elements in $R$ such that $X_{p}$ is algebraically independent over $A$ and $A_{p}\left[X_{p}\right]=R_{p}$. As in the previous argument there is an $f_{p} \in A_{p}\left[X_{p}\right]$ such that $R\left[1 / f_{p}\right]=A\left[X_{p}, 1 / f_{p}\right]$. Thus, with the possible exception of the finite set of essential valuations of $f_{p}$, every essential valuation of $R$ is an essential valuation of $A_{p}\left[X_{p}\right]$. If we set $S=A-p$, we have $\left(A\left[x_{p}\right]\right)_{S}=A_{p}\left[X_{p}\right]=R_{S}=R_{p}$. Thus the essential valuations of $R$ which are not 
essential for $A\left[X_{p}\right]$ are lost when we localize $R$ at the multiplicative system $S$. Therefore there is an $s \in S$ such that every essential valuation of $f$ is positive on $s$ and hence $A\left[X_{p}, 1 / s\right]=R[1 / s]$. Thus for every prime $p \subset A$ there is an $s_{p}$ $\notin p$ such that $R\left[1 / s_{p}\right]$ is an affine ring over $A$. Since the ideal generated by the set of $s_{p}$ is contained in no prime ideal of $A$, it must be that there is a finite set of the $s_{p}$ which generate the unit ideal. Changing notation somewhat, let $\left\{s_{1}, \ldots\right.$, $\left.s_{n}\right\}$ denote this set with $p_{i}$ the prime corresponding to $s_{i}$ and $X_{i}$ a corresponding set of indeterminates such that $A_{p_{i}}\left[X_{i}\right]=R_{p_{i}}$. We claim $R=A\left[X_{1}, \cdots, X_{n}\right]$. We clearly have $A\left[X_{1}, \ldots, X_{n}\right] \subseteq R$. Since each is an $A$-module, to check for equality we need only check to see that they are locally equal as $A$-modules. Let $P$ be any prime of $A$. Then for some $i, s_{i} \notin P$. Thus $R_{p}=\left(R\left[1 / s_{i}\right]\right)_{p}=\left(A\left[X_{i}, 1 / s_{i}\right]\right)_{p}$ $\subseteq\left(A\left[X_{1}, \cdots, X_{n}\right]\right)_{p} \subseteq R_{p}$. Thus $A\left[X_{1}, \cdots, X_{n}\right]=R$ and we are finished. To see that $R$ is Krull, let $P$ be any prime of $A$. Then we have seen that there is an $s$ $\notin P$ such that $R[1 / s]=A\left[X_{p}, 1 / s\right]$. Since $A\left[X_{p}\right]$ is Krull, $R[1 / s]$ is Krull. The element $s$ is in only finitely many height one primes of $D$, say $q_{1}, \cdots, q_{g}$. For any height one prime $q$, either $q$ is one of $q_{1}, \cdots, q_{g}$ or $R[1 / s] \subseteq R_{q}$. Thus $R[1 / s] \cap \bigcap_{i=1}^{g} R_{q_{i}} \subseteq \bigcap_{\mathrm{ht} q=1} R_{q}=R$ by (2.26). So $R=R[1 / s] \cap \bigcap_{i=1}^{g} R_{q_{i}}$. Therefore, $R$ is the intersection of a finite number of Krull rings and it is therefore Krull.( $\left.{ }^{1}\right)$

(3.4) If $R$ is locally a polynomial ring over a domain $A$ and is contained in an affine domain over $A$, then $R$ is a UFD if and only if $A$ is a UFD.

Proof. This follows immediately from (3.1) and (3.4).

(3.5) Suppose $A$ is a noetherian integrally closed domain and $R$ is locally a polynomial ring over $A$. If $R$ is contained in an affine ring over $A$ then $R$ is an affine ring over $A$. In particular, $R$ is noetherian.

Proof. Since such an $A$ is Krull we need only observe that we can reduce to the case where $R$ is contained in an affine domain. This follows immediately from the following.

(3.6) Suppose that $R$ is a domain which is contained in a noetherian ring $A$. Then there is some beight zero prime $P$ of $A$ such that $P \cap R=(0)$.

Proof. Since $A$ is noetherian, there are only a finite number of height zero primes in $A$, say $P_{1}, \ldots, P_{n}$. For each $i$, let $Y_{i}=P_{i} \cap R$. Since $\cap P_{i}$ contains only nilpotents, $\cap Y_{i}=(0)$. Thus there is some $i$ such that $P_{i}=0^{i}$.

It seems reasonable to expect that if $R$ is locally a polynomial ring over a noetherian domain $A$, and $R$ is contained in an affine ring over $A$, then $R$ should

(1) This result can be strengthened to the extent that one need only assume that $R$ is locally affine over $A$. 
be noetherian. However, at this time we are unable to decide if this is the case. We can, however make a number of observations:

Recall that a prime $P$ is said to be a weak-Bourbaki prime of an ideal $A$ if there exists an $x$ such that $P$ is minimal among the primes which contain $(A: x)$.

(3.7) If $R$ is locally polynomial over a noetherian ring $A$ in a finite number of indeterminates, then the following are equivalent.

(i) $R$ is noetherian.

(ii) Every finitely generated ideal of $R$ bas only a finite number of weakBourbaki prime divisors.

(iii) There exist elements $s_{1}, \cdots, s_{n} \in A$ such that $R\left[1 / s_{i}\right]$ is noetherian for each $i$ and the ideal $\left(s_{1}, \cdots, s_{n}\right)$ bas depth zero.

Proof. If $R$ is noetherian, then the weak-Bourbaki prime divisors are precisely the usual associated primes. Thus every ideal has only finitely many weak-Bourbaki primes and (i) implies (ii). Since $R$ is locally polynomial over a noetherian ring in a finite number of indeterminates it is locally noetherian. Thus (ii) implies (i) by [HO, (1.4)]. Clearly (iii) follows from (i) by taking the generators of any maximal ideal of $A$. Suppose then that (iii) is satisfied. Since $A$ is noetherian, ( $s_{1}$, $\cdots, s_{n}$ ) has a finite number of associated primes. Since this ideal has depth zero, they must all be maximal, call them $m_{1}, \cdots, m_{t}$. Then these are the only primes of $A$ which contain $\left(s_{1}, \cdots, s_{n}\right)$. Let $I$ be an ideal of $R$. In each of the localizations at $m_{1}, \cdots, m_{t}$ and $s_{1}, \cdots, s_{n}$ the extension of $I$ can be generated by a finite number of elements from $I$. Let $I^{\prime}$ be the ideal of $R$ generated by these elements. Then $I^{\prime}$ is a finitely generated ideal which is contained in $I$. If $p$ is any prime of $R$, then either $p$ is equal to one of the $m_{i}$ 's or there is an $s_{i}$ which is not contained in $p$. In either case, $I \otimes A_{p}=I^{\prime} \otimes A_{p}$ by the choice of generators for $I^{\prime}$. Thus $I=I^{\prime}$.

(3.8) Suppose that $R$ is both locally polynomial over a noetherian domain $D$ and contained in an affine ring over $D$. Let $R_{(0)}=K\left[X_{0}\right]$ where $X_{0}$ is a set of indeterminates over $D$ and $K$ is the quotient field of $D$. Then there is an $s \in D$ such that $R[1 / s]=D\left[1 / s, X_{0}\right]$.

Proof. By (3.6) we can assume that $R$ is contained in an affine domain over $D$. Suppose that $D\left[\xi_{1}, \cdots, \xi_{n}\right]$ is a domain which contains $R$. Then $D\left[\xi_{1}, \cdots, \xi_{n}\right]$ is an affine domain over $D\left[X_{0}\right]$ and by $[\mathbf{N}, \mathbf{l} \mathbf{r},(14.4)]$ there is an $f \in D\left[X_{0}\right]$ such that $D\left[\xi_{1}, \ldots, \xi_{n}, 1 / f\right]$ is a finite module over $D\left[X_{0}, 1 / f\right]$. Since $D\left[X_{0}, 1 / f\right]$ is noetherian, $R[1 / f]$ is also a finite module over $D\left[X_{0}, 1 / f\right]$. Thus there is some $b \in D\left[X_{0}, 1 / f\right]$ such that $b R \subseteq D\left[X_{0}, 1 / f\right]$. Now $b=g / f^{t}$ for some integer $t$ and some $g \in D\left[X_{0}\right]$, thus $f^{t} b \in D\left[X_{0}\right]$. Let $s$ be the product of the coefficients of $f^{t} b$ and the coefficients of $f$. Then 


$$
f^{t} b R[1 / s] \subseteq D\left[X_{0}, 1 / s, 1 / f\right] \cap K\left[X_{0}\right]
$$

Let $b \in K\left[X_{0}\right]$ be contained in $D\left[X_{0}, 1 / s, 1 / f\right] . b=b^{\prime} / f^{m}$ for some integer $m$ and some $b^{\prime} \in D\left[X_{0}, 1 / s\right]$. Thus $f^{m} b=b^{\prime}$. Taking content over $D[1 / s], A_{f}=D[1 / s]$ and $A_{b^{\prime}} \subseteq D[1 / s]$. Since $A_{f}=D[1 / s]$,

$$
A_{f} m_{b}=A_{f} A_{b}=D[1 / s] A_{b}=A_{b} .
$$

Thus $A_{b}=A_{b^{\prime}} \subseteq D[1 / s]$ and $b \in D\left[1 / s, X_{0}\right]$. Therefore,

$$
f^{t} b R[1 / s] \subseteq D\left[1 / s, X_{0}\right]
$$

and since the content of $f^{t} b$ is also the unit ideal we have $R[1 / s] \subseteq D\left[1 / s, X_{0}\right]$. The reverse inclusion is clear.

(3.9) If $D$ is a one-dimensional noetherian domain and $R$ is botb locally polynomial over $D$ and contained in an affine ring over $D$, then $R$ is an affine ring over $D$.

Proof. Using the notation of (3.8), we have $R[1 / s]=D\left[1 / s, X_{0}\right]$ for some $s \epsilon$ $D$. Let $p_{1}, \cdots, p_{n}$ be the primes of $D$ which contain $s$. For each $i$, let $X_{i}$ represent the finite set of indeterminates over $D$ which are contained in $R$ such that $R_{p_{i}}=D_{p_{i}}\left[X_{i}\right]$. Let $R^{\prime}=D\left[X_{0}, X_{1}, \cdots, X_{n}\right]$. If $p$ is any prime of $D$ not containing $s$, then $R_{p}=R_{p}^{\prime}$. If $p$ contains $s$, then $R_{p}=R_{p}^{\prime}$ by the choice of generators. Since $R$ and $R^{\prime}$ are locally equal, we have $R=R^{\prime}$.

An integral domain $D^{\prime}$ is said to be an almost finite integral extension of a domain $D$ if $D^{\prime}$ is integral over $D$ and the quotient field of $D^{\prime}$ is finite over the quotient field of $D$. A domain $D$ is said to have the finiteness condition for integral extensions if every almost finite integral extension of $D$ is a finite extension. If $D$ is a noetherian domain which satisfies the finiteness condition for integral extensions then so does a polynomial ring over $D$ in a finite number of indeterminates. This result can be partially generalized to locally polynomial rings.

(3.10) Suppose that $D$ is noetherian domain with finiteness condition for integral extensions. If $R$ is locally polynomial and affine over $D$, then $R$ also satis. fies the finiteness condition for integral extensions.

Proof. Suppose that $R=D\left[r_{1}, \cdots, r_{n}\right]$. Let $F$ be the quotient field of $R$ and let $L$ be a finite algebraic extension of $F$. Since $R$ is noetherian, we need only show that $R^{*}$, the integral closure of $R$ in $L$, is a finite extension of $R$. We can choose elements $x_{1}, \cdots, x_{m} \in R^{*}$ such that $L=F\left(x_{1}, \cdots, x_{m}\right)$. Let $R^{\prime}=$ $R\left[x_{1}, \cdots, x_{m}\right]=D\left[r_{1}, \cdots, r_{n}, x_{1}, \cdots, x_{m}\right]$. If $P$ is a prime of $R^{\prime}$ and $S=D=$ $(P \cap D)$, we have

$$
D_{S} \subseteq R_{S}=D_{S}\left[t_{1}, \cdots, t_{k}\right] \subseteq R_{S}^{\prime}=D_{S}\left[t_{1}, \cdots, t_{k}, x_{1}, \cdots, x_{m}\right] \subseteq R_{S}^{*},
$$


where $t_{1}, \cdots, t_{k}$ is an algebraically independent set over $D_{S}$. Since $D$ has the finiteness condition, $D_{S}$ and hence $D_{S}\left[t_{1}, \ldots, t_{k}\right]$ have the finiteness condition $\left[\mathbf{N}, 1 \mathbf{r},(35.1)\right.$ and (35.2.]. Thus $R_{S}^{*}$ is a finite extension of $D_{S}\left[t_{1}, \cdots, t_{k}\right]$ and is therefore a finite module over $R_{S}^{\prime}$. Then $R_{P}^{*}$ is a finite module over $R_{P}^{\prime}$. This must be true for any prime $P$ of $R^{\prime}$, so $R^{*}$ must be a finite module over $R[\mathbf{N}, 1 \mathbf{r}$, (35.3)], Thus $R$ has the finiteness condition for integral extensions.

A ring $A$ is said to be pseudogeometric if $A$ is noetherian and for every prime $p$ of $A, A / p$ has the finiteness condition for integral extensions.

(3.11) Suppose that $R$ is locally polynomial over a pseudogeometric domain $A$ and contained in an affine ring over $A$. Then $R$ is an affine ring over $A$.

Proof. Suppose $R \subseteq A\left[\xi_{1}, \cdots, \xi_{n}\right]=A^{\prime}$. By (3.6) we can assume that $A^{\prime}$ is a domain. By (1.12) the derived normal ring $\bar{R}$ of $R$ is locally polynomial over the derived normal ring $\bar{A}$ of $A$. By [N, lr, (36.6)], the derived normal ring $\overline{A^{\prime}}$ of $A^{\prime}$ is a finite module over $A^{\prime}$ and is therefore affine over $\bar{A}$. Now $[\mathbf{N}, \mathbf{l r},(36.5)]$ shows that $\bar{A}$ is pseudogeometric and hence Krull, consequently by (3.1) $\bar{R}$ is Krull and it is affine over $\bar{A}$ by (3.3). Thus there are elements $r_{1}, \ldots, r_{m}$ and $s_{1}, \ldots$, $s_{m}$ in $R$ such that

$$
\bar{R}=\bar{A}\left[r_{1} / s_{1}, \cdots, r_{m} / s_{m}\right] .
$$

Let $t_{1}, \cdots, t_{k}$ be the coefficients of the equations of integral dependence of the $r_{i} / s_{i}^{\prime} s$ over $R$. Let

$$
R^{\prime}=A\left[r_{1}, \cdots, r_{m}, s_{1}, \cdots, s_{m^{\prime}} t_{1}, \cdots, t_{k}\right] .
$$

Then $R^{\prime} \subseteq R$ and $\overline{R^{\prime}}=\bar{R}$. Since $R^{\prime}$ is affine over $A$, it is pseudogeometric by $[\mathbf{N}, \mathbf{l r},(36.5)] . \bar{R}$ is therefore a finite module over $R^{\prime}$ and since $R^{\prime}$ is noetherian, the submodule $R$ is also finite over $R^{\prime}$. Thus $R$ is affine over $A$.

We close this article with a closer look at the type of example with which we opened: rings which are locally polynomial rings in one variable over a principal ideal domain.

(3.12) If $R$ is locally a polynomial ring in one variable over the principal ideal domain $A$, then $R$ is an affine ring over $A$ if and only if $R$ is a polynomial ring over $A$.

Proof. Let $K$ denote the quotient field of $A$ and $R_{(0)}=K[X]$. Suppose $R=$ $A\left[X_{1}, \cdots, X_{n}\right]$. There is no loss in assuming that the $X_{i}$ are local generators for the primes $p_{1}, \cdots, p_{n}$ (i.e. $A_{p_{i}}\left[X_{i}\right]=R_{p_{i}}$ ).

Moreover, we may assume $R_{q}=A_{q}[X]$ for all primes $q \notin\left\{p_{1}, \cdots, p_{n}\right\}$. Consider the semilocal ring $A_{\left(P_{1}, \cdots, P_{n}\right)}\left[X_{1}, \cdots, X_{n}\right]$. This is locally a polynomial ring. Hence by (1.22) $A_{\left(P_{1}, \cdots, P_{n}\right)}\left[X_{1}, \cdots, X_{n}\right]=A_{\left(p_{1}, \cdots, p_{n}\right)}[\theta]$ for some $\theta$. Thus 
$R=A[X, \theta]$. We write $\theta=(a+b X) / s$ where $a, b, s \in A$ and no divisor of $s$ divides both $a$ and $b$.

We first observe that $(s, b)=1$. For suppose $\pi$ is some prime element which divides both $b$ and $s$. Then $a+b X \in \pi R$ and if $\pi \mid b$ then $\pi \mid a$ in $R$. Thus $\pi$ divides $a$ in $A$, in contradiction to the assumption that no prime divisor of $s$ divides both $a$ and $b$. We now search for an element $T$ such that $R \subseteq A[T]$. Since $(b, s)=1$, by the Chinese remainder theorem we can find an element $C_{1}$ in $A$ such that $C_{3}=\left(a+b C_{1}\right) / s$ is in $A$. Set $S=C_{2}$ and $b=C_{4}, a=-C_{1} / s, \beta=1 / s$. Then $A[X,(a+b X) / s] \subseteq A[\alpha+\beta X]$ since $X=C_{1}+C_{2}(\alpha+\beta X),(a+b X) / s=C_{3}+$ $C_{4}(\alpha+\beta X)$. Thus $A \subseteq R \subseteq A[T]$ and in view of (1.19) there is a fractionary ideal Q⿱ 2 of $A$ and a transcendental $t$ over $A$ such that $R=A[\mathcal{U} t]$. Since $A$ is a PID, $\mathcal{U}$ is principal, say $\mathfrak{Q}=\lambda A$. Thus $R=A[\lambda t]$ and $R$ is a polynomial ring over $A$.

(3.13) Suppose $R$ is locally a polynomial ring over the Krull ring A. Let $K$ denote the quotient field of $A$ and $X$ a set of elements in $R$ which are algebraically independent over $K$ and such that $R_{(0)}=K[X]$. Then $R$ is an affine ring over $A$ if and only if there is an $f \in A[X]$ sucb that $A[X, 1 / f]=R[1 / f]$. Thus $R$ is an affine ring over $A$ if and only if almost every essential valuation for $A[X]$ is es. sential for $R$ and almost every essential valuation of $R$ is essential for $A[X]$.

The proof of this is essentially the same as the proof of (3.5).

If we put (3.12) and (3.13) together we can conclude the following:

(3.14) If $R$ is locally polynomial in one variable over the principal ideal domain $A$ then these are equivalent:

(i) $R$ is a polynomial ring over $A$.

(ii) $R$ is an affine ring over $A$.

(iii) $R$ is contained in an affine ring over $A$.

(iv) If $K$ denotes the quotient field of $A$ and $X$ is an element of $R$ such that $R_{(0)}=K[X]$, then with at most a finite number of exceptions, every essential valuation for $R$ is essential for $A[X]$ and every essential valuation for $A[X]$ is essential for $R$.

The hypothesis of PID cannot be relaxed to Dedekind. For let $X$ be an indeterminate over a Dedekind domain $D$. If $\mathcal{U}$ is a nonprincipal ideal of $D$ then $R=$ $D[U X]$ is an affine ring over $D$ which is locally a polynomial ring. However $R$ is not a polynomial ring over $D$ (see [AEH, Example (3.2)]).

With regard to (3.12) a natural question is: If $R$ is locally a polynomial ring in one variable over the PID $A$ and $R$ is noetherian, is $R$ affine over $A$ ? The answer is negative for we are able to construct a counterexample with the integers as the ground ring.

(3.15) Example. A ring $R$ which is locally a polynomial ring in one variable over the integers $Z$ and such that 
(1) $R$ is noetherian.

(2) $R$ is a UFD.

(3) $R$ is not an affine ring over $Z$.

Construction. First, if $V$ is a valuation ring with quotient field $K$ and $X$ is an indeterminate over $V$ then by $V(X)$ we denote the trivial, or canonical extension of $V$ to $K(X)$. This is the valuation which assigns to $f \in K[X]$ the minimum of the $V$-valuations of its nonzero coefficients [ZS, II, p. 85]. With this notation one always has $V[X]=V(X) \cap K[X]$. Now let $X$ be an indeterminate over $Z$ and let $\left\{\left(p_{i}, m_{i}\right)\right\}_{i=1}^{\infty}$ be a collection of ordered pairs of positive integers such that $p_{i}$ is prime and no nonzero element of $Z[X]$ is in more than finitely many of the maximal ideals $M_{i}=\left(x-m_{i}, p_{i}\right) \in Z[X]$. To construct such a family one could let $\left\{f_{i}\right\}_{i=1}^{\infty}$ be a denumeration of the nonzero elements of $Z[X]$ and $g_{j}=\Pi_{i=1}^{j} f_{i}$. Choose the pair $\left(p_{j}, m_{j}\right)$ as follows:

(i) $g_{j} \neq 0\left(P_{j}\right)$,

(ii) $\operatorname{deg} g_{j}<P_{j}$,

(iii) $m_{j}$ is not a root of $g_{i}$ modulo $P_{j}$.

Then the collection $\left\{\left(m_{i}, P_{i}\right)\right\}_{i=1}^{\infty}$ has the desired property.

Let $\Delta=\bigcup_{i=1}^{\infty}\left\{P_{i}\right\}$, now let $\theta_{j}=\left(X-m_{j}\right) / P_{j}$ and set $R=Z\left[\left\{\theta_{j}\right\}_{j=1}^{\infty}\right]$. Then $Z[X] \subset R$ and if $p$ is any prime in $Z$ then

$$
R_{p}= \begin{cases}Z_{p}[X] & \text { if } p \notin \Delta \\ Z_{P_{j}}\left[\theta_{j}\right] & \text { if } p=P_{j} \in \Delta .\end{cases}
$$

Thus $R$ is locally a polynomial ring over $Z$. Now we claim that $R$ is noetherian. Since $R$ is an overring of $Z[X]$ it suffices to prove that $R$ is Krull in view of a theorem of Heinzer [H].

By (1.10) we can write

$$
\begin{aligned}
R & =\left(\bigcap_{P_{j} \in \Delta} Z_{P_{j}}\left[\theta_{j}\right]\right) \cap\left(\bigcap_{p \notin \Delta} Z_{p}[X]\right) \\
& =\left(\bigcap_{P_{i} \in \Delta} Z_{P_{j}}\left(\theta_{j}\right) \cap Q\left[\theta_{j}\right]\right) \cap\left(\bigcap_{p \notin \Delta}\left[Z_{p}(X) \cap Q[X]\right]\right) \\
& =\left[\bigcap_{P_{j} \in \Delta} Z_{P_{j}}\left(\theta_{j}\right) \cap\left[\bigcap_{p \notin \Delta} Z_{p}(X)\right] \cap Q[X]\right] \\
& =R_{1} \cap R_{2} \cap Q[X] .
\end{aligned}
$$

Since the finite intersection of Krull rings is Krull it suffices to see that each of the se three rings is Krull. Obviously $Q[X]$ is Krull.

The ring $R_{2}$ is Krull since it is an intersection of essential valuations of the Krull ring $Z[X]$. To see that $R_{1}$ is Krull it suffices to see that no nonzero element of $R_{1}$ has positive value in more than finitely many of the valuations $Z_{P_{j}}\left(\theta_{j}\right)$. The center of the valuation ring $Z_{P_{j}}\left(\theta_{j}\right)$ on $Z[X]$ is the ideal $\left(x-m_{j}, P_{j}\right)$ and no 
nonzero element of $Z[X]$ is in more than finitely many of these maximal ideals. Thus $R_{1}$ and hence $R$ is Krull. This ring cannot be an affine ring over $Z$ by (3.14) since more than a finite number of its essential valuations (the $Z_{P_{j}}\left(\theta_{j}\right)$ ) are not essential for $Z[X]$. The ring $R$ is a UFD by (3.1)

\section{BIBLIOGRAPHY}

[AEH] S. Abhyankar, P. Eakin and W. Heinzer, On the uniqueness of the coefficient ring in a polynomial ring, J. Algebra (to appear).

[B] N. Bourbaki, Elements de mathématique. Fasc. XXVII. Algëbre commutative. chaps. 1, 2, Actualités Sci. Irdust., no. 1290, Hermann, Paris, 1961. MR 36 \#146.

[Ch] S. U. Chase, Direct products of modules, Trans. Amer. Math. Soc. 97 (1960), 457473. MR $22 \# 11017$.

[Cl] L. Claborn, Every abelian group is a class group, Pacific J. Math 18 (1966), 219-222. MR $33 \# 4085$.

[Co] P. M. Cohn, Bezout rings and their subrings, Proc. Cambridge Philos. Soc. 64 (1968), 251-264. MR 36 \#5117.

[Ce] D. Coleman and E. E. Enochs, Isomorphic polynomial rings, Proc. Amer. Math. Soc. 27 (1971), 247-252. MR $42 \# 7686$.

[EK] P. Eakin and K. Kubota, $A$ note on the uniqueness of rings of coefficients in polynomial rings, Proc. Amer. Math. Soc. 32 (1972), 333-341.

[GJ] L. Gillman and M. Jerison, Rings of continuous functions, Van Nostrand, Princeton, N. J., 1960. MR $22 \# 6994$.

[G] R. Gilmer, Multiplicative ideal theory, Queen's Papers in Pure and Appl. Math., no. 12, Queen's University, Kingston, Ont., 1968. MR 37 \#5198.

[H] W. Heinzer, On Krull overrings of a Noetherian domain, Proc. Amer. Math. Soc. 22 (1969), 217-222. MR $40 \# 7235$.

[Ja] P. Jaffard, Théorie de la dimension dans les anneaux de polynomes, Mémor. Sci. Math., fasc. 146, Gauthier-Villars, Paris, 1960. MR 22 \#8038.

[Je] C. U. Jensen, A remark on flat and projéctive modules, Canad. J. Math. 18 (1966), 943-949. MR $33 \# 7375$.

[K] I. Kaplansky, Commutative rings, Allyn and Bacon, Boston, Mass., 1970. MR 40 $\# 7234$.

[N, 1r] M. Nagata, Local rings, Interscience Tracts in Pure and Appl. Math., no. 13, Interscience, New York, 1962. MR 27 \#5790.

$[\mathrm{N}, \mathrm{fgr}]$ - . A theorem on finite generation of a ring, Nagoya Math, J. 27 (1966), 193-205. MR $33 \# 2670$.

[OR] J. Ohm and D. Rush, The finiteness of I when $R[X] I$ is flat, Trans. Amer. Math. Soc. (to appear).

[OR, cma] - Content modules and algebras (to appear).

[S] P. Samuel, Lectures on unique factorization domains, Tata Institute of Fundamental Research Lectures on Math., no. 30, Tata Institute of Fundamental Research, Bombay, 1964. MR 35 \#5428.

[V] W. V. Vasconcelos, On finitely generated flat modules, Trans. Amer. Math. Soc. 138 (1969), 505-512. MR 39\#199.

[ZS] O. Zariski and P. Samuel, Commutative algebra. Vol. II, University Series in Higher Math., Van Nostrand, Princeton, N. J., 1960. MR 22 \#11006.

DEPARTMENT OF MATHEMATICS, UNIVERSITY OF KENTUCKY, LEXINGTON, KENTUCKY 40506

DEPARTMENT OF MATHEMATICS, KING COLLEGE, BRISTOL, TENNESSEE 37620 